\title{
Measurements in wrinkled flame by laser Doppler velocimetry and laser tomography
}

\author{
R. HADEF (corresponding author) \\ BP 297 Institut de Génie Mécanique, Centre Universitaire Larbi Ben M’Hidi \\ 04000 Oum El Bouaghi - Algérie \\ Email: rhadef@ rocketmail.com \\ G. SEARBY* and J. QUINARD* \\ Laboratoire de Recherche en Combustion, Université de Provence, 13397 Marseille - France
}

\begin{abstract}
This paper deals with the application of laser Doppler velocimetry when associated with laser tomography to weakly stretched propagating downwards flames. Its main objective is the determination of the Markstein number representing sensitivity to strain and curvature of the premixed wrinkled flames. The measured quantities are the flow field velocity of the unburned gas, the normal flame velocity and the amplitude of the flame front. The experimental data are obtained for methane and propane laminar flames stabilized in a sinusoidally (spatially) modulated steady flow. An original method, using the tomography laser technique is developed to stabilize intrinsically the flame.

The results obtained are in good agreements with those found in the literature, confirming the dependence of Markstein number values on value of Lewis number.
\end{abstract}

Keywords: Laser Doppler velocimetry - laser tomography - Premixed wrinkled flame Markstein number.

\section{Introduction}

Today combustion science may be considered from two convergent points of view. The first one is represented by physicists who are able to give sophisticated models where the combustion mechanisms (i.e. diffusion processes and chemical reactions) are taken into account as well as the hydrodynamics $[1,2]$. The second point of view deals with the evolution of aircraft gas turbine combustion technology over the past forty years which is impressive, and whose recent developments caused significant shifts in development emphasis toward combustion technology [3]. For example, in addition to the necessary improvements to increase thrust/weight ratio, new concepts and technology improvements are necessary to satisfy recent exhaust pollutant regulations $[4,5]$. Thus, in order to validate new theories or to allow figures to numerical simulations, or to control the characteristics of an engine, accurate tools are developed with the advent of the lasers [6]. As a matter of fact, most of the relevant information is obtained by noninvasive, local and instantaneous measurements. These needs can be satisfied by using optical techniques.

It is well know that combustion regime in several practical systems is turbulent and the flame-flow interaction is described in terms of total front surface and local flame structure. The local flame properties defined by the displacement speed and reaction rate depend on local hydrodynamic strain and curvature effects as mentioned in asymptotic theories [7,8]. Furthermore, the flame response to an incoming flow depends strongly on the Markstein number noted $M a$, which characterises the local behaviour of the flame front.

The aim of this work is mainly concerned with presenting how cattering of laser light provides one solution for measuring in reacting flow. The paper describes the development of a combined laser Doppler velocimetry (LDV) [15] with a laser tomography (TM) [16] scattering systems with the objective of stabilizing intrinsically a wrinkled flame, and measuring the crucial parameter, $M a$. The flame form is established in a steady periodic shear flow.

This parameter may be found in the evolution equation of the turbulent front flame [7]. It controls the variation magnitude in the normal flame speed associated with the local value of flame stretch produced by either the front curvature or the divergence of the external flow field [8]:

$$
\frac{U_{N} ? U_{L}}{U_{L}} \text { ? Ma } \stackrel{?}{?} \frac{d}{R} ? \frac{1}{U_{L}} \stackrel{?}{n} . ? ? \stackrel{?}{U} ? \stackrel{?}{n} \stackrel{?}{?}
$$

where $? ? \dot{U} ?$ is the rate of strain tensor of the upstream flow, evaluated at the reaction zone. In its original introduction [9], this number was defined as a length $L$ with $M a ? L / d$.

This relation has been used to measure locally the Ma value [10], but the small local change in burning velocity as well as the difficulty of extrapolating flow velocities to the position of the reaction zone, gave discouraging results, presenting a high uncertainly in the value of $M a$.

An expression of the value of $M a$ has been given in the limit of high activation energy, small strain, and 
a one-step reaction [11]. Later, this calculation has been extended [12] to include the temperature dependence of diffusivities and led to:

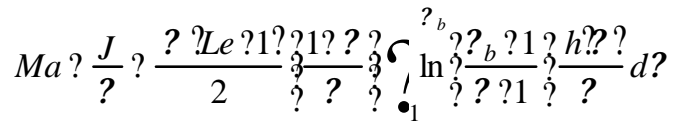

where the quantity $J$ is the integral of the temperature dependence of the diffusion coefficients defined as:

$$
J ? \overbrace{\bullet_{1}}^{?_{\mathrm{b}}} \frac{h(?)}{?} d ?
$$

The above integrals can be evaluated using standard data for gaseous nitrogen. Taking a burned gas temperature of $T_{b} ? 1670 \mathrm{~K}$ and ? 0.825 , one finds:

$$
\text { Ma? 4.1 ? 0.41? ?Le?1? }
$$

(4)

It can be seen that the Markstein number is a positive quantity [13] except for lean hydrogen flames where the Lewis number may be sufficiently small. If the Lewis number is close to unity, the Markstein number is expected to be close to 4 . The usefulness of Eq. 4 ceases here because the concept of the Lewis number is only valid for a one-step reaction.

Another theoretical analysis has been performed using a reduced three-step reaction of a weakly strained stoichiometric methane-air flame with the simplified assumption that diffusion coefficients are independent of temperature [14]. This has led to an analytical relationship expression for the Markstein number, which is formally similar to Eq. 2. The expression gives a value of $M a ? 2.23$ for methane. A less restrictive numerical calculation has also been performed and has given $M a$ ? 3.4.

\section{Optical diagnostics}

\subsection{Laser Doppler velocimetry}

The LDV technique is based on Lorenz-Mie scattering diagnostics that provide strong signal from particles in the flows. It is well described in the references [17-20]. In this technique a beam splitter is used to split the laser beam into two beams of equal intensity. Then the laser beams intersect, using a focusing lens, at one point called the probe volume (Fig. 1). A fringe pattern of bright (high intensity) and dark (low intensity) lines is created at the point of intersection. The space between the fringes, bright lines, $i$, is constant and related to the laser beam wavelength as:

$$
i ? \frac{?}{2 \sin ? / 2}
$$

The flow is seeded with particles that scatter light when crossing the fringes. Monitoring the Mie scattered light from a particle crossing the probe volume using a photodetector, e.g. photomultiplier tube (PMT) or photodiode, leads to a LDV signal, burst. The signal is then filtered (Fig. 2) and used to provide the change in the frequency of the particles (Doppler shift), $F_{D}$. This provides the time of a particle crossing two successive fringes, $T_{D}$. Thus, based on this time and the calculated distance of the fringe spacing, the velocity component $U_{\text {? }}$ (perpendicular to fringes) of the particle, within the control volume can be calculated through the relation:

$$
U_{?} ? F_{D} i
$$

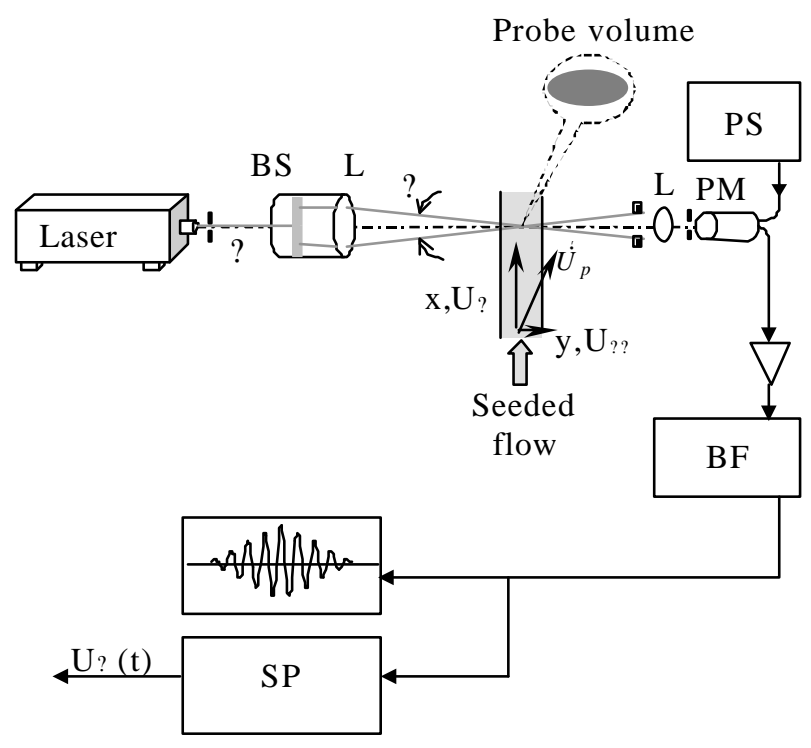

BS : Beamsplitter

PM: Photomultiplier

BF: Bandpass filter

PS: Power supply

L : Focusing lens

SP: Signal processor

Figure 1- Optical arrangement for the LDV measurements

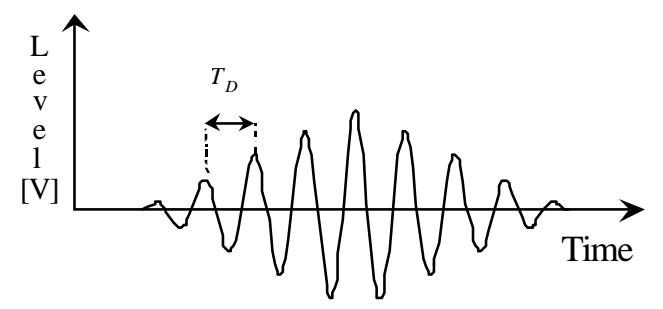

Figure 2- Bandpass filtered Doppler burst signal

In turbulent medium, flow velocity measurements need a careful choice of both the size and density (if possible) of the particles. The general motion equation for a sphere is given by [20,21]:

$$
\begin{aligned}
& \frac{1}{6} ? d_{p}^{3} ?_{p} \frac{d \dot{U}_{p}}{d t} ?-3 ? ? d_{p} \stackrel{?}{U}_{r} ? \frac{?}{6} d_{p}^{3} ? \frac{d \stackrel{U}{U}^{t}}{d t} \\
& ? \frac{?}{12} d_{p}^{3} ? \frac{d \stackrel{U}{U}_{r}}{d t} ? \frac{3}{2} d_{p}^{2} \sqrt{? ? ?} \overbrace{t_{t o}}^{\frac{d \dot{U}_{r}}{d t^{\prime}}} \frac{\mathrm{dt}^{\prime}}{\sqrt{\mathrm{t}-\mathrm{t}^{\prime}}}
\end{aligned}
$$


This equation has been studied extensively [23]. In laser velocimetry, a good approximation of this relation may be obtained if Stokes drag term is only considered. The time response of a particle to the variation of the velocity is:

$$
?_{p} ? \frac{1}{18} \frac{d_{p}^{2} ?_{p}}{?}
$$

A representative example of the values taken by the time response may be given by assuming a $1-\mu \mathrm{m}$ oil droplet in air at 1 atmosphere. For this case, $?_{p} ? 3 \mu s$ and the frequency for which it follows the flow within $3 \mathrm{~dB}$ is $50 \mathrm{kHz}$. In some circumstances, the settling velocity should also be taken into account. For the above example, this velocity is about $30 \mu \mathrm{ms}^{-1}$. Finally, it is worth while noticing that the LDV technique can lead to measuring both velocity particles as well as flow velocity when properly seeded.

\subsection{Laser Tomography}

Laser tomography in combustion provides a visualization of flame fronts (Fig. 3).

A vertical beam of laser light crosses the reaction zone. When a fresh gas mixture is seeded with a fine mist of $1-\mu \mathrm{m}$ oil particles, a laser beam passing through the mixture is visualized due to the fact that the light is strongly scattered in all directions (regardless of the anisotropy of scattering which has no influence). On some flame surface ? $(y, z, t)$, the gases burn in a thin zone of about $0.1 \mathrm{~mm}$ in which the oil droplets vaporise at an isotherm of approximately $600 \mathrm{~K}$ and burn. A striking feature of the absence of droplets in burnt gases is the vanishing of light scattering. The laser beam cannot be visualized in the burnt mixture gas (transparent gas), and the position of the frontier between the bright and dark regions is expressed by ? $(y, z, t)$.The local position of the front is detected by imaging the laser sheet onto a slit.

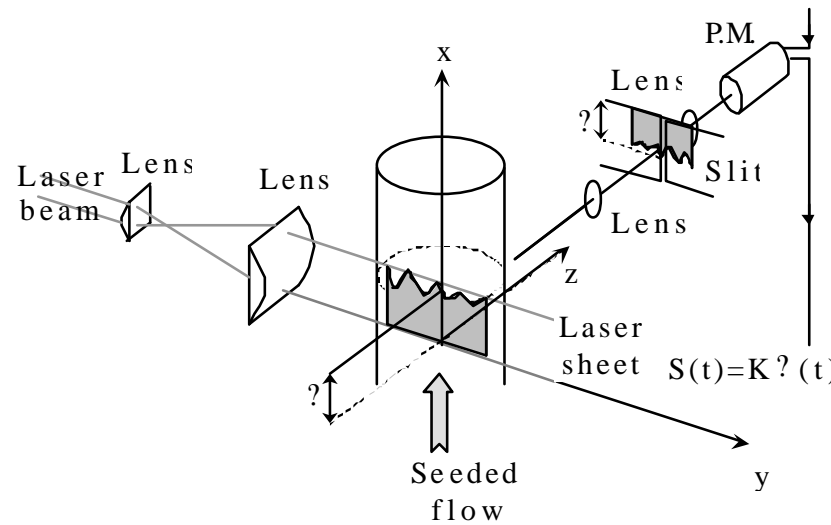

Figure 3- Schematic arrangement for obtaining the instantaneous flame front position and velocity
The light flux passing through this latter is then detected by a photodiode, which gives a signal proportional to the flame position. Taking the derivation of this signal, one may easily obtain the instantaneous fluctuating part of the velocity of the flame front ???/?t?.

\section{Experimental}

\subsection{The Burner}

A schematic representation of the burner is shown on Fig. 4. Its head is rectangular in cross section with sides equalling 200x $80 \mathrm{~mm}$; those walls are made of Pyrex glass. The reactive premixed flow enters from below after laminarisation in a setting chamber. An initial "top-hat" velocity profile is ensured by using grids and a convergent section placed between the chamber and the burner head.

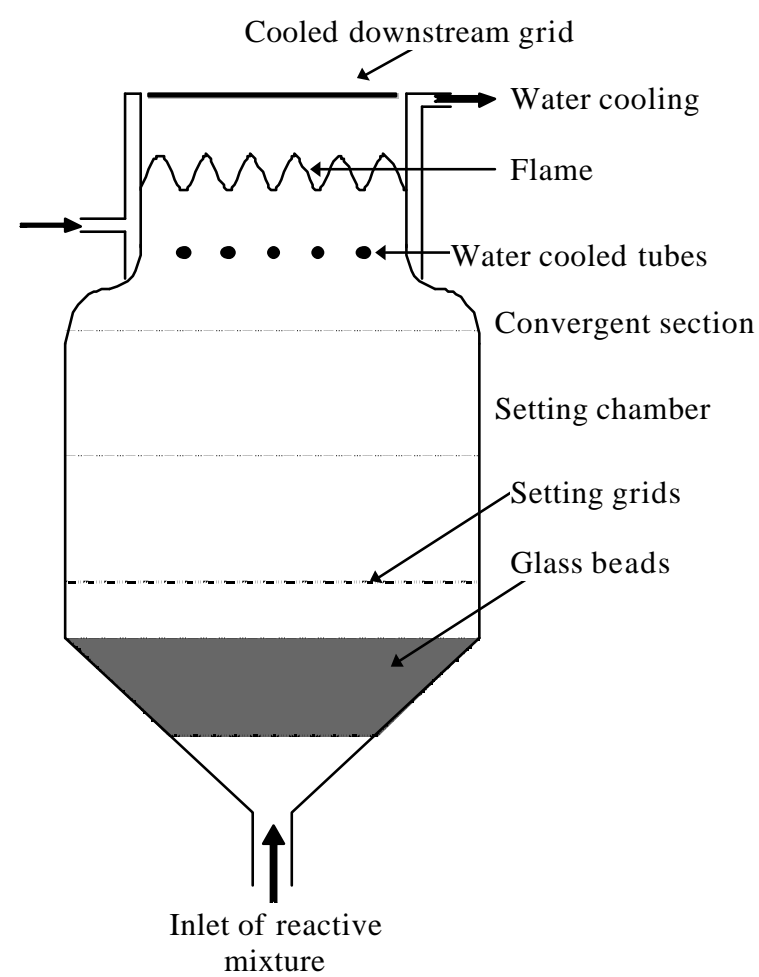

Figure 4- Schematic of premixed wrinkled flame burner

At the exit from the convergent section a number of equidistant tubes (horizontal spacing 15, 20, 25, or $30 \mathrm{~mm}$ ) are placed in the flow (Photo 1). The external diameter of the tubes $(4 \mathrm{~mm})$ is such that the flow around them is laminar $(\operatorname{Re}<25)$. These tubes create a laminar perturbation in the velocity profile that is close to a set of periodic negative delta functions just behind them. As this shear flow is convected downstream, the high-wavenumber components are rapidly attenuated by viscous forces, and $80 \mathrm{~mm}$ from the tubes the flow profile becomes closely sinusoidal with about $5 \%$ to $10 \%$ 
of first harmonic content (higher harmonics are completely negligible). The peak-to-peak velocity modulation is found to be equal $15 \%$ to $40 \%$ of the mean flow velocity, depending on the tube spacing. In order to eliminate the thermal convection problems that would be caused by heating of the tubes by the flame radiation and from the burner downstream walls, the tubes are stabilized at the temperature of the upstream gas by internal water circulation. A water-cooled grid maintained at $85^{\circ} \mathrm{C}$ and placed at the exit from the burner decouples the hot flow from the cold ambient air. Namely, the temperature walls of the settling chamber and the burner head are controlled with independent water bath circulators to prevent the presence of thermal boundary layers.

As shown in photo 2, the flame takes the form of a two-dimensional sinusoidal sheet with wavelength of wrinkling equal to the spacing of the bars. The amplitude of the wrinkling given by Eq. 9 depends both on the ability of the flame to modify its local burning velocity to match that of the incoming, and on the modification of the incoming flow created by the deviation of the streamlines through the flame front (hydrodynamic feedback). It should be remembered that the flame is stable only at relatively low burning velocities [24] where the Darrieus-Landau hydrodynamic instability is sufficiently weak to be balanced by the stabilizing effects of gravity and diffusion processes.

\subsection{Stabilization of the flame}

In this study, we aim to obtain an unattached flame whose average position is constant with respect to the burner. This position is then the one where the mean flame and the mean flow velocities are equal. This situation can be achieved by an active stabilization loop (Fig. 5): the spatially averaged position of the front ? $(y, z, t)$ is optically detected by the tomography laser on a photodiode. The delivered signal $S(t)$ is then used to control with the servo-loop the flow rate of fresh gas coming into the burner [10]. The reactive mixture whose composition is known to a precision of $1 \%$ is produced by metering air, fuel, and nitrogen in appropriate proportions through sonic throat nozzles. In order to permit regulation of the flow without change in composition, the mixture is produced in excess and the unused fraction is vented to the atmosphere.

\subsection{The optical system}

A schematic diagram of the optical and signal processing systems is shown in Fig. 6. A Spectra Physics 164 4:Watt argon-ion laser is used as the light source. The beam coming out the laser is separated on colour: $?_{1}=488 \mathrm{~nm}$ for the LDV and $?_{2}=514.5 \mathrm{~nm}$ for the laser tomography. The LDV beam is split into two beams. A $300 \mathrm{~mm}$ focal length focused both beams to a diameter of about

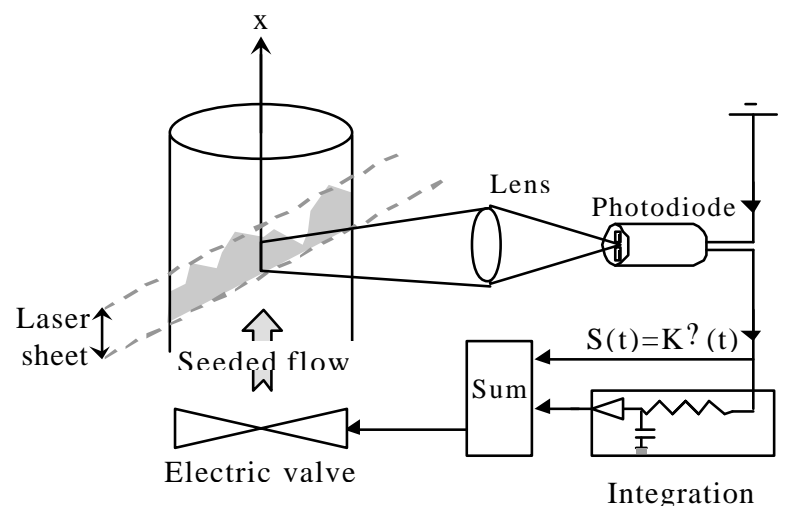

Figure 5 - A flame stabilization device

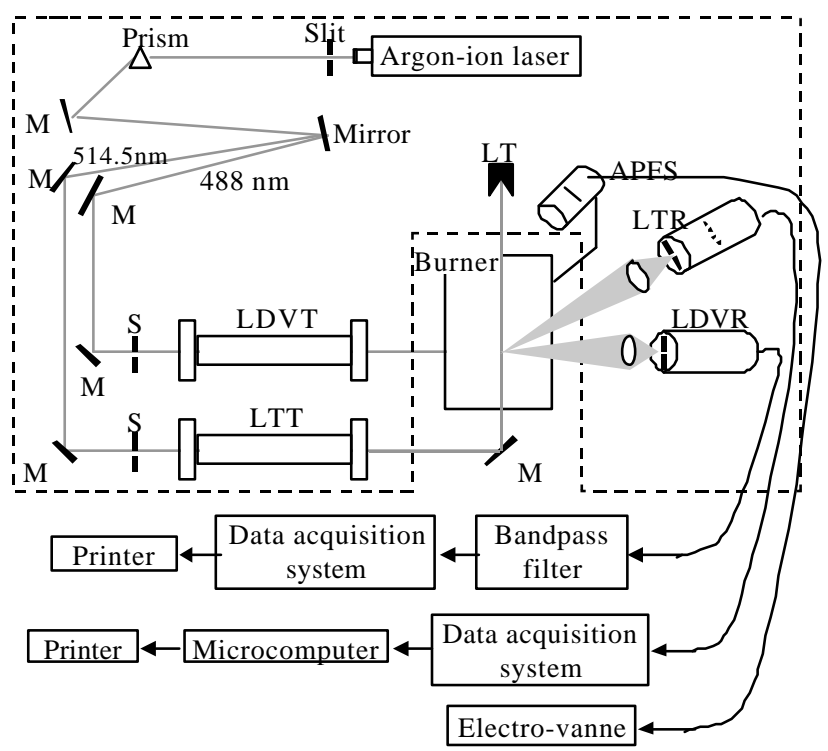

APFS : array of photodiodes for the flame stabilization LDVT : laser Doppler velocimetry transmission

LDVR : laser Doppler velocimetry receiving

LTT : laser tomography transmission

LTR : laser tomography receiving

LT : light trap

Figure 6- Optical, signal processing and data acquisition systems

$0.19 \mathrm{~mm}$ corresponding to a fringe spacing of $1.27 \mu \mathrm{m}$. A $50 \mathrm{~mm} \mathrm{f} / 3.5$ lens is used to collect the on-axis forward the scattered light and in order to focus it on the photomultiplier tube through $1 \mathrm{x}$ $0.1 \mathrm{~mm}$ slit. The collected light is filtered by a $3 \mathrm{~nm}$ bandpass, $488 \mathrm{~nm}$ interference filter placed behind the slit. The filtered light then falls onto the cathode of 14 dynodes photomultiplier. The signal from the photomultiplier is first amplified and then forwarded to the acquisition module.

The tomography beam is expanded by a $10 \mathrm{X}$-beam expander, thus forming a vertical sheet $(1 \times 20 \mathrm{~mm})$. This latter crosses the combustion zone, the 
droplets in the unburned gas scattered the laser light and the burned gas is transparent. The local position of the front is detected by imaging the laser sheet onto a vertical CCD array of 256 photodiodes. The spatial resolution is fixed at 77 pixels $/ \mathrm{mm}$ (1pixel=13? $\mathrm{m}$ ). The contour of the flame front captured at several times is extracted with an edge detection algorithm, using thresholding procedure. A microcomputer monitors the CCD array and determines the flame position in real time $(8$ measures par second).

\subsection{The Experimental Method}

Markstein number is obtained from the measurement of the global response of the flame front, assumed to be planar on average, to a known incident flow field using the results of Searby and Clavin [25] who have presented a complete analysis of a wrinkled flame front propagating downwards in a nonhomogeneous and/or unsteady incoming flow field. The calculations were carried out for an arbitrary value of the gas expansion ratio and with temperature dependent transport coefficients, representing the case where the flame is intrinsically stable. Explicit result is expressed in Fourier space, and relates the amplitude, $A(k, ?)$, of a Fourier component of the flame front wrinkling to the amplitude, $U_{e}(k, ?)$, of a Fourier component of the fluctuation of the longitudinal component of the flow field. More precisely, $U_{e}(k, ?)$ is a flow field fluctuation that would have been present in the absence of the flame, and is to be evaluated at the position of the reaction zone. One may quote the result of these authors simplified to the case of a steady nonuniform flow (that is for the case ? ? 0) and retaining only the dominant order terms:

$\frac{A(k)}{U_{e}(k)}$ ?

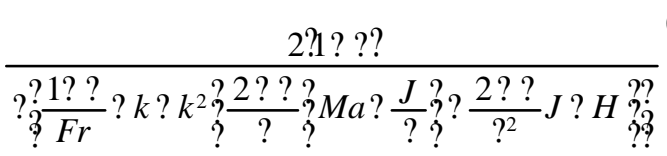

with

$$
H ? h_{b} ? ? 2 \operatorname{Pr} ? 1 ?^{\sigma_{1}}{ }^{?_{b}} \frac{h_{b} ? h(?)}{\left(?_{b} ? 1\right)} d ?
$$

The reduced amplitude $A(k)$ is equal to the absolute flame front amplitude ? divided by the flame thickness $d$ and the reduced flow velocity $U_{e}(k)$ is equal to the ratio of the excited flow velocity to the la minar flame velocity $U_{L}$. A typical recording of the shape of the flame front and a longitudinal velocity profile is shown in Fig. 7 and the equation 9 has been used to evaluate the
Markstein number of premixed flame in a sinusoidally modulated steady shear flow.

The wrinkled flame shape is measured by the laser tomography technique described above. The contour representing the position of the flame front is Fourier analyzed to obtain the amplitude of the wrinkling at the wavelength of excitation.

The flow field reduced velocity $U_{e}(k)$ of Eq. 9 is the Fourier component of the flow field that would have been present in the absence of flame, but measured at the position of the reaction zone, that is, excluding the effects of the induced flow field $U_{i}(k)$ produced by the flame. It not possible to measure this quantity directly because the thermal convection effects, arising from heating of the rods by the thermal flame radiation and downstream grid, are sufficient to slightly perturb the hot flow.

The following procedure was used to measure $U_{e}(k)$ :

The longitudinal velocity profile, as shown in Fig. 7, is measured at a number of different distances upstream from the front. This velocity profile is then Fourier analyzed and the appropriate Fourier component plotted as a function of the upstream distance as shown in Fig. 8. Far upstream, the shear modulation experiences slow exponential decay due to viscous damping and possible thermal buoyancy effects. Closer to the flame, the induced flow field causes the total flow modulation to vary rapidly on the scale of ${ }_{e} / 2$ ? . The flow field velocity $U_{e}(k)$ is found extrapolating the incident flow field to the position of the flame as shown in Fig. 8. Because this incident flow field is only slowly changing, there is no need to know the position of the reaction zone with high precision.

Methane flame, $?=1.0, \mathrm{U}_{\mathrm{L}}=9.6 \mathrm{~cm} / \mathrm{s}$

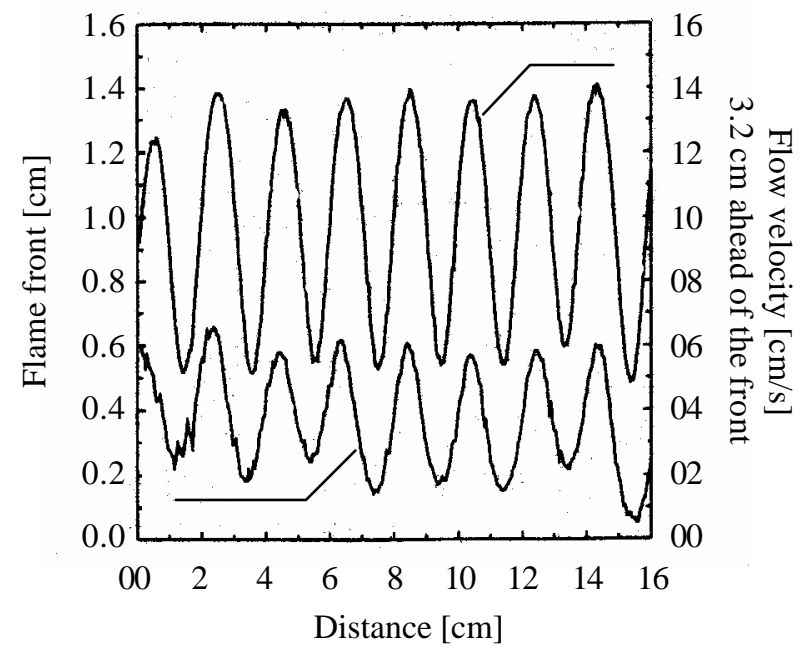

Figure 7 - Typical measured shape of flame front(TL) and longitudinal velocity (LDV) profile 
The Froude number is obtained from a measurement of the laminar burning velocity $U_{L}$ of the same mixture with a nonmodulated flow (with the exception of the rods) using the LDV. The flow velocity profile is found to be flat to within $5 \%$

Methane flame, ? $=0.7, \mathrm{U}_{\mathrm{L}}=8.4 \mathrm{~cm} / \mathrm{s}$

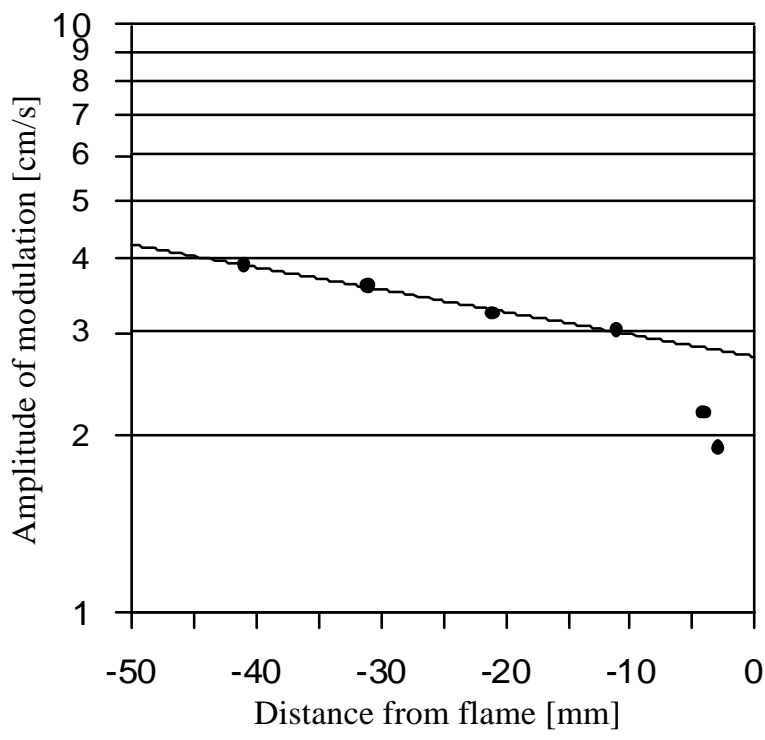

Figure 8- Amplitude of modulation (LDV) of incoming flow velocity,

over a rectangular $180 \times 60 \mathrm{~mm}$, and the level of residual turbulence less than $1 \%$. It is possible to obtain planar flames flat to within $1 \%$ over the full 180x60 mm central part of the front. The stabilizing effect of gravity keeps the flame flat at long wavelengths and improves the flow profile close to the flame.

The burning velocity of the flame is measured by recording the longitudinal gas velocity along a line perpendicular to the front. It is relatively easy to determine $U_{L}$ in these experiments because the upstream velocity gradient is ideally zero, and in practice is so small to be always negligible $\left(<0.1 \mathrm{~s}^{-}\right.$ ${ }^{1}$ ). The burning velocity could be determined in this way to better than 0.5 [26].

The other quantities appearing in Eq. 9 are known or easily calculated. The reduced burned gas temperature $?_{b}$ is calculated for each mixture assuming adiabatic combustion with a heat of reaction of $460 \mathrm{kcal} / \mathrm{mol}$ for propane and 173 $\mathrm{kcal} / \mathrm{mol}$ for methane. The burned gas temperature $T_{b}$ is also determined experimentally from measurements of the burnt gas velocity for certain mixtures [24] confirming these values for the heats of reaction. The other quantities Pr, ?, $h_{b}, J$ and $H$ are calculated using standard data [27] for oxygen, nitrogen, propane, and methane and from the knowledge of the unburned as well as the burned gas temperatures. The integral $J$ and
$H$ are evaluated numerically. Their exact numerical values depend slightly on the mixture composition, but typical values are:

$$
\begin{gathered}
\operatorname{Pr} \text { ? 0.69, ? } 0.82, h_{?} ? 3.2, \\
J ? 3.3 \text {, and } H ? 4.27
\end{gathered}
$$

\section{Results}

Measurements are made on methane flames at two different equivalence ratios:? ? 0.70 and ? ?1.0. The burning velocity is varied independently of the equivalent ratio by changing the dilution ? with nitrogen. It is expected that the Markstein number would depend only on the equivalent ratio and not on nitrogen dilution, at least for small changes in the burning velocity. The flame response is measured at a number of different burning speeds ranging from close to extinction $\left(U_{L} ? 7 \mathrm{~cm} / \mathrm{s}\right)$, to close to the instability threshold $\left(U_{L} ? 10 \mathrm{~cm} / \mathrm{s}\right)$. The results are plotted in Fig. 9 as a function of the Froude number along with lines of constant $M a$ calculated from Eq. 9. Measurements were also carried out at the same equivalence ratios but for a constant burning velocity as a function of the wavelength of excitation ?? $e_{e}$ ?1.5, 2.0, 2.5 and $3.0 \mathrm{~cm}$ ?. The results are shown in Fig. 10 again along with lines of constant $M a$ calculated from Eq. 9. It can be seen that the response of the flame as functions of both the Froude number and a reduced wavenumber are reasonably well represented by Eq. 9 and lead to the values Ma? 3.1? 0.3 at ? 0.7 and Ma? 3.6 ? 0.3 at ? ? 1.0 for methane

Similar measurements were made on propane flames as a function of the burning velocity for the two equivalence ratios ? 0.52 and ? 1.0 . The results are shown in Fig. 11. Again, the evolution of the response as a function of the Froude number is well described by Eq. 9 and leads to the values Ma? 4.5? 0.5 at ? ? 0.52 and Ma? $3.9 ? 0.5$ at ? ? 1.0 for propane.

For both methane and propane flames, the Markstein number is positive and close to 4 for a stoichiometric mixture (Fig. 12). According to the Eq. 4 and the linear dependence of the effective Lewis number ? $L e ? 1$ ? on the equivalence ratio value (Fig. 13), it is not surprising that the methane flame have a Markstein number, which increases with composition and propane flames have a Ma value which drops rapidly with? value.

It can be seen that propane flames $(e=1.4)$ are more influenced by stretched effects with a Markstein number greater than methane $(L e=0.85)$, while the flame response to flow divergence and curvature depends strongly on the Lewis number characterised by the ratio of molecular diffusivity to thermal diffusivity. For non-unity Lewis numbers, differential diffusion of heat and mass species 
results in a certain sensitivity of the local flame structure to strain and curvature.

\section{Conclusion}

Two laser diagnostic methods for combustion studies were presented. Namely, they have been used to determine $M a$ from the global response of the flame to a nonuniform flow. The flow incoming is a steady parallel flow, with a longitudinal gas velocity modulated sinusoidally in one transverse direction with a wavelength $?_{e}$ that can be chosen experimentally.

We conclude that this method is a tedious task involving several experimental measurements (flame shape, flow velocities). The values obtained for $M a$ are found to be quite compatible with those obtained from the stability limits [10]. This is not really surprising since the calculation of $M a$ basically relies on the use of the same theoretical analysis of flame structure. However, for the same equivalence ratio, it is possible in this case to carry out measurements at different burning velocities and spatial frequencies within the region of planar flame stability. It is found that the dependence of the global response on both velocity and wavenumber are reasonably well represented by the theoretical relations, and this is a strongs element in favor of their validity. Thus it also allows confidence on the results obtained from the use of the stability.

Rogg and Peters [14] have performed a theoretical and a numerical analysis of a weakly strained stoichiometric methane flame using a reduced three-step reaction with two intermediate species. They found a numerical result of $M a ? 3.4$ which may be considered to be in good agreement with our experiment value of $M a$ ? 3.7 ? 0.4 .

B. Renou et al. [28] have recently reported such measurements of the flame curvature and local displacement flame speed ???/?t? on freelypropagating turbulent premixed flames simultaneously for methane and propane/air flames, without a strain. The values obtained are close to those found by this method.

\section{Acknowledgements}

We gratefully acknowledge the financial support of this research by the C.E.E. (Contrat $N^{\circ}$ EN3E0084-F). We wish to thank Pr. Paul Clavin for his many helpful discussions 
Photo 1. Bars of modulation

Photo 2. Propane flame front (bleu) stabilized in the burner The LT sheet (green) as well as the LDV beams (bright) are clearly visible 
a)- Methane $\quad ?=0.7, \mathrm{~T}_{\mathrm{b}}=1270 \mathrm{~K}+\mathrm{T}_{0}$

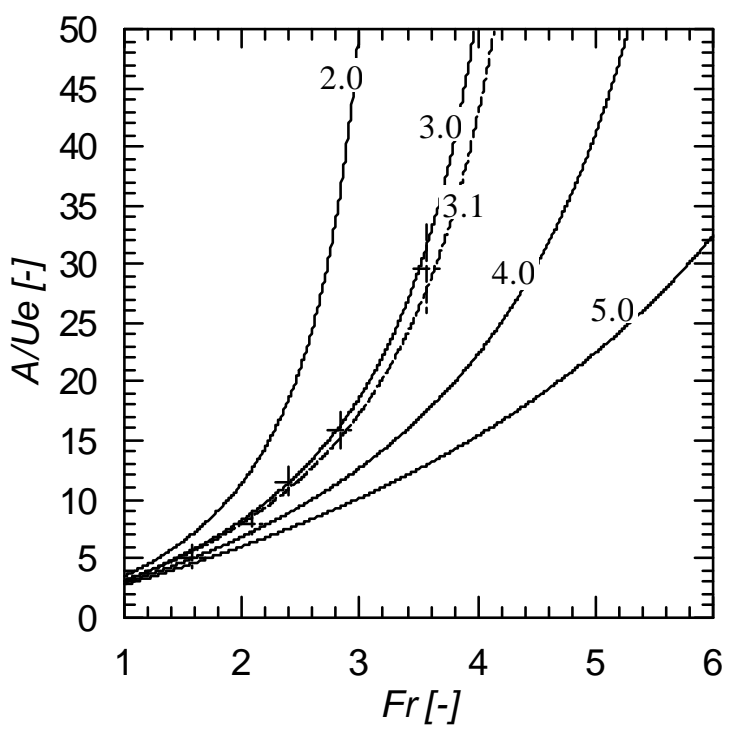

b)- Methane $\quad ?=1.0, \mathrm{~T}_{\mathrm{b}}=1390 \mathrm{~K}+\mathrm{T}_{0}$

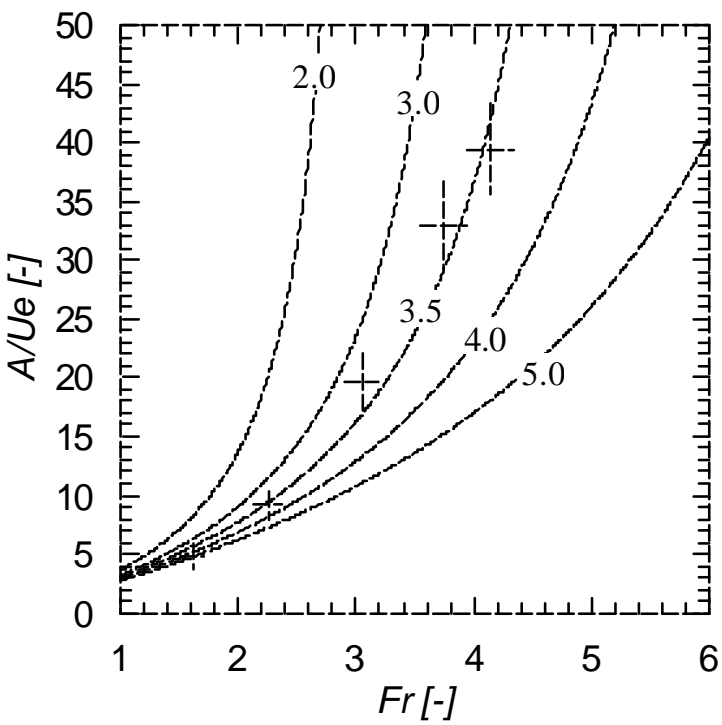

Figure 9- Reduced response of methane flame to the periodic shear flow as a function of the Froude number, Fr. Dotted line is best fit to experimental points

a)- Methane $?=0.7, ?=0.176, \mathrm{~T}_{\mathrm{b}}=1250 \mathrm{~K}+\mathrm{T}_{0}$

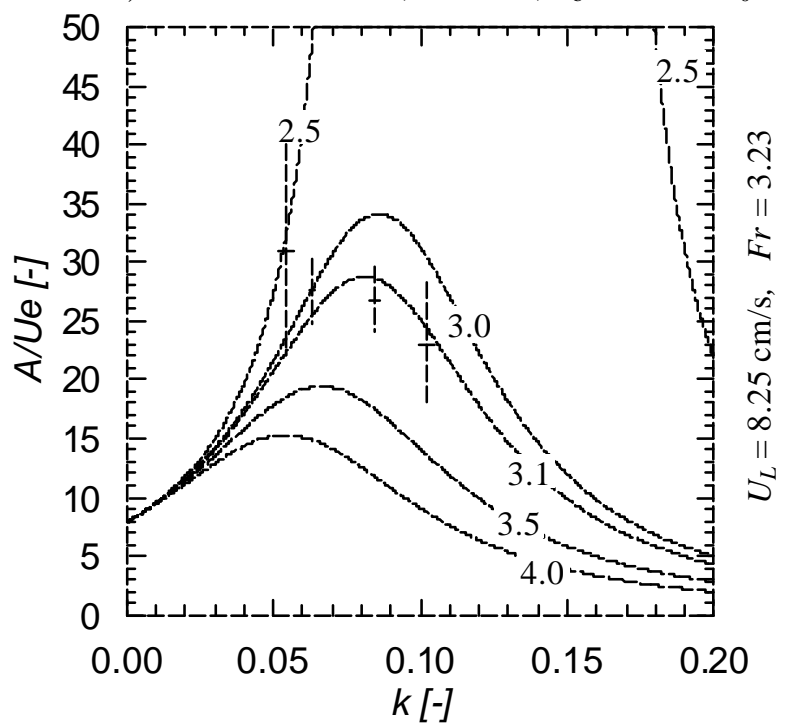

b)- Methane $?=1.0, ?=0.140, \mathrm{~T}_{\mathrm{b}}=1380 \mathrm{~K}+\mathrm{T}_{0}$

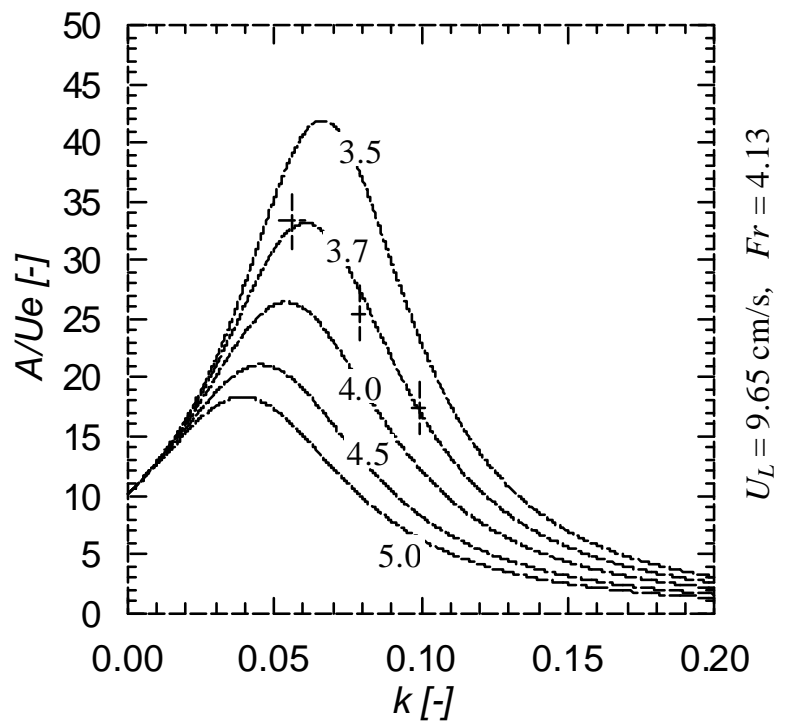

Figure 10- Reduced response of methane flame to the periodic shear flow as a function of the reduced wavenumber, $k$. Dotted line is best fit to experimental points 
a)- Propane $\quad ?=0.52, \quad \mathrm{~T}_{\mathrm{b}}=1200 \mathrm{~K}+\mathrm{T}_{0}$

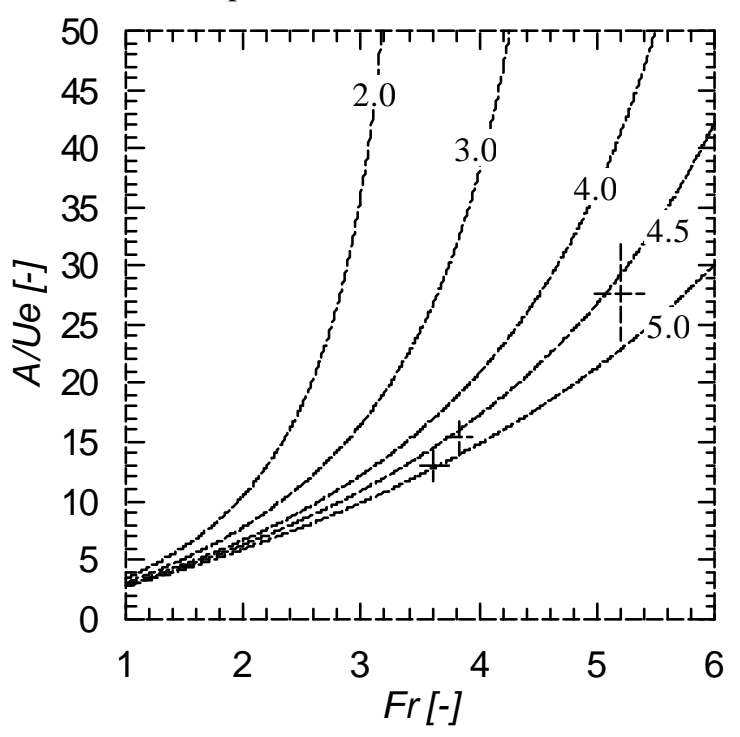

b)- Propane $\quad ?=1.0, \mathrm{~T}_{\mathrm{b}}=1400 \mathrm{~K}+\mathrm{T}_{0}$

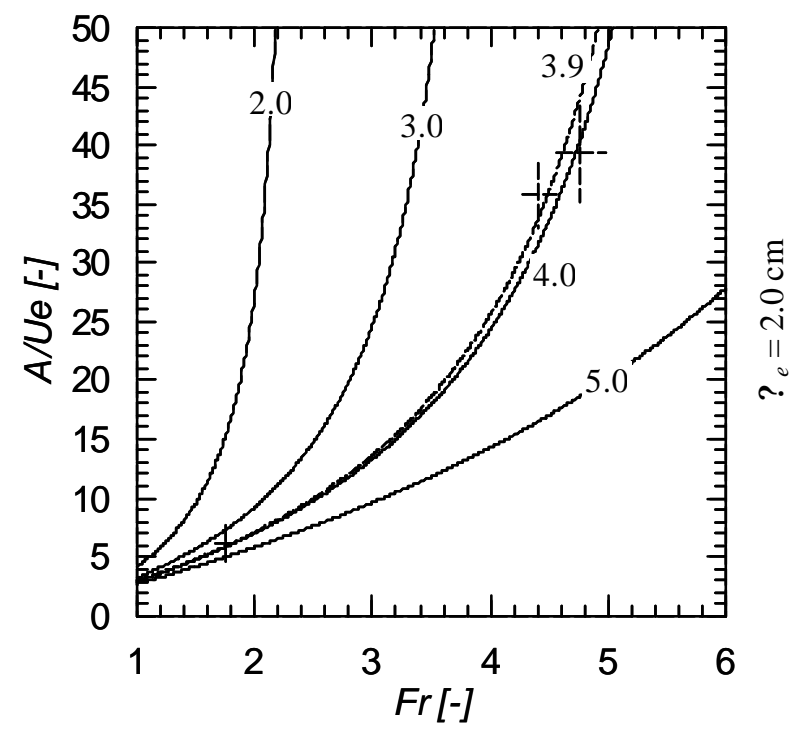

Figure 11- Reduced response of propane flame to the periodic shear flow as a function of the Froude number, Fr. Dotted line is best fit to experimental points

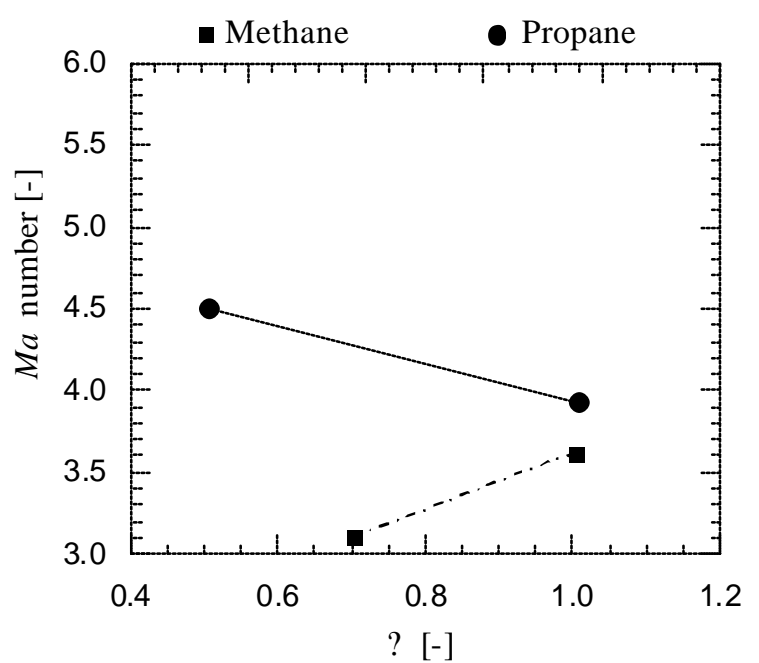

Figure 12- Values of the Markstein number as a function of the equivalence ratio

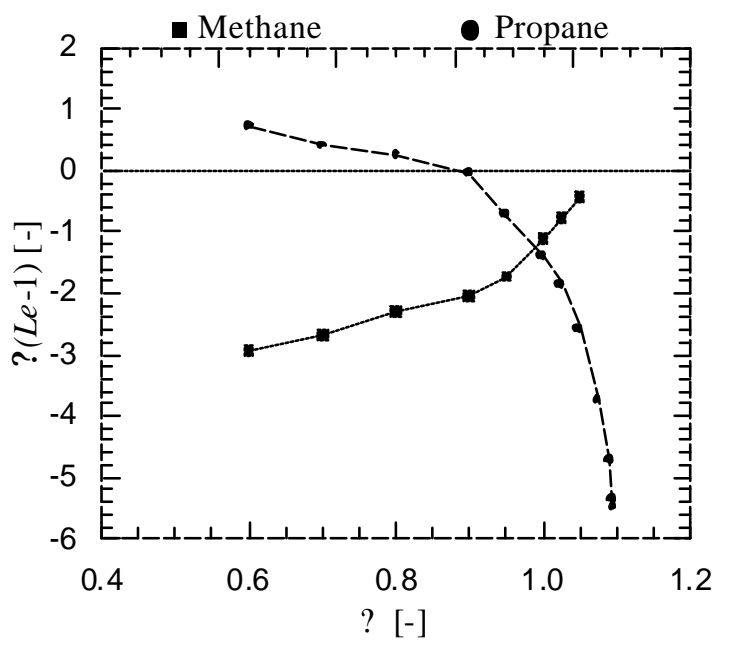

Figure 13- Values of the effective Lewis number as function of the equivalence ratio [24] 


\section{Nomenclature}

$A(k)$ amplitude of Fourier component of flame wrinkling, reduced by the flame thickness

$d \quad$ flame thickness, $\mathrm{D}_{\mathrm{th}} / \mathrm{U}_{\mathrm{L}}$

$d_{p} \quad$ particle diameter

$d_{r} \quad$ rod diameter

$D_{m o l}$ molecular diffusivity of unburned gas

$D_{t h} \quad$ thermal diffusivity of unburned gas

$D_{t h b} \quad$ thermal diffusivity of burned gas

$E$ activation energy of chemical reaction

$F_{D} \quad$ frequency Doppler burst

$g \quad$ acceleration of gravity

$h($ ?) temperature dependence of diffusivity, $D_{t h} / D_{\text {tho }}$

$h_{b} \quad h\left(?_{b}\right)$

$i \quad$ fringe spacing

$J \quad$ integral involving temperature dependence of diffusivities

$K \quad$ coefficient of proportionality

$k \quad$ reduced wavenumber, $2 ? d / ?_{e}$

$L \quad$ Markstein length, $L / d$

$\dot{n}$ unit vector normal to the front directed towards the unburned gases

$R$ gas constant or mean curvature radius of flame front

$S(t) \quad$ amplitude of the analogue signal

$t \quad$ time

$T$ temperature

$T_{o} \quad$ temperature of unburned gas

$T_{b} \quad$ temperature of burned gas

$T_{D} \quad$ period of Doppler burst

$\dot{U} \quad$ flow field velocity of gas

$U \quad$ component of longitudinal gas velocity

$U_{e}(k)$ amplitude of Fourier component of longitudinal gas velocity of the exciting gas field

$U_{i}(k)$ amplitude of Fourier component of longitudinal gas velocity of the induced gas field

$U_{L} \quad$ normal burning velocity of planar flame

$U_{N} \quad$ normal burning velocity of stretched flame

$\dot{U}_{p} \quad$ field velocity of particle

$U_{r} \quad$ relative particle velocity, $\dot{U}_{p} ? \dot{U}$

$U_{\text {? }} \quad$ velocity component perpendicular to fringes

$U_{/ /} \quad$ velocity component (parallel to fringes) of particle

$V \quad$ component of transversal gas velocity

$(\mathrm{x}, \mathrm{y}, \mathrm{z})$ coordinates for space

? equivalence ratio

$?_{e} \quad$ wavelength of wrinkling

? amplitude of the wrinkled flame

? Zeldovich number, $\mathrm{E}\left(\mathrm{T}_{\mathrm{b}}-\mathrm{T}_{\mathrm{o}}\right) / \mathrm{RT}_{\mathrm{b}} / \mathrm{T}_{\mathrm{b}}$

? dilution of the mixture, $\mathrm{O}_{2} /\left(\mathrm{O}_{2}+\mathrm{N}_{2}\right)$

? gas expansion parameter, $\left(\mathrm{T}_{\mathrm{b}}-\mathrm{T}_{\mathrm{o}}\right) / \mathrm{T}_{\mathrm{b}}$
? dynamic viscosity

? cinematic viscosity

? angle between the laser beams

? wavelength of the laser beam

? reduced temperature, $\mathrm{T} / \mathrm{T}_{\mathrm{o}}$

$?_{\mathrm{b}} \quad$ reduced temperature of burned gas, $\mathrm{T}_{\mathrm{b}} / \mathrm{T}_{\mathrm{o}}$

? density of the gas

$?$ p density of the particle

? $\quad$ time particle response to flow acceleration

? frequency

Le Lewis number, $D_{t h} / D_{m o l}$

$M a \quad$ Markstein number, $L / d$

Pr Prandt number, $\mathrm{D}_{\mathrm{th}} /$ ?

Fr Froude number, $U_{L}^{2} /(g d)$

Re Reynolds number, $U d_{r} /$ ?

\section{References}

[1] H. P. Schmidt, P. Habisreuther and W. Leuckel (1998) "A model for calculating heat release in premixed turbulent flames" Comb. Flame $113,79$.

[2] P. Clavin and F. A. Williams (1982) "Effects of molecular diffusion and thermal expansion on the structure and dynamics of premixed flames in turbulent flows of large scale and low intensity" J. Fluid Mech. 116, 251.

[3] R. Hadef, K. Merkle, B. Lenze and W. Leuckel (2000) “An experimental study of air-blast atomizer spray flames" Journal of the Institute if Energy-London 494, 50

[4] K. Merkle, A. Ament, B. Lenze, W. Leuckel and R. Hadef (1999) "Untersuchungen zum Stabiltätsverhalten einer Airblast-Zertäuberdüse " VDI-Berrichte 1492, 449.

[5] T. Terasaki and S. Hayashi (1996) "The effects of Fuel-air mixing on $\mathrm{NO}_{\mathrm{x}}$ Formation in non-premixed swirl burners " $26^{\text {th }}$ Symp. Inter. on Combustion, 2733.

[6] R. Hadef, K. Merkle, B. Lenze and W. Leuckel (2000) "Flow measurements in a liquid fuel flame" accepted for the $26^{\text {th }}$ Cong. Inter. Meeting on Chem. Eng. Env. Protect. and Biotech. ACHEMA 2000 (Frankfurt)

[7] P. Clavin (1985) "Dynamic behaviour of premixed flame fronts in laminar and turbulent flows" P. Prog. Energy Combust. Sci. 11, 1.

[8] Williams F. A. (1985) "Combustion theory" $2^{\text {nd }}$ Edition, the Benjamin/Cummings Publishing Company, Inc. ,415.

[9] G. H. Markstein (1951) "Experimental and theoretical studies of flame front instability" J. Aerospace Sci., 18, 199.

[10] R. Hadef (1987) "Mesure du nombre de Markstein dans une flamme plissée" Thèse de Doctorat de l'Université de Provence, France. 
[11] P. Clavin and F. A. Williams (1979) "Theory of premixed flame propagation in large scale turbulence" J. Fluid Mech. 90, 589.

[12] P. Clavin and P. Garcia (1983) "The influence of the temperature dependence of diffusivities on the dynamics of flame fronts" J. Méc. Théor. Appl. 2, 245.

[13] A. Linan and P. Clavin (1987) "Premixed flames with non-branching chain reactions (structure and dynamics) " Combust. Flame 70, 137.

[14] B. Rogg and N. Peters (2000) "The asymptotic stucture of weakly strained stoichiometric methane-air flame" to be pubmished in Comb. Flame

[15] Y. Yeh and H. Z. Cummins (1964) App. Phys. Let. 4-176.

[16] L. Boyer (1980) Laser tomographic method for flame front movement studies" Comb. Flame $39,321$.

[17] L. E. Drain (1980) "Laser Doppler technique" Wiley, Chichester.

[18] F. Durst, A. Melling and J. H. Whitelaw "Principles and practice of laser Doppler Anemometry" Academic Press, New York (1981)

[19] R. J. Andrean "Laser Velocimetry Fluid Mechanics Measurements" Ed. R. J. Goldstein 287311, Hemisphere, Springer-Verlag, Berlin (1983)

[20] A. Haddad, L. Khezzar, M. H. Benhamza, R. Hadef and H. Satha (1999) "La vélocimétrie laser et ses applications en hydrodynamique" $\mathrm{fr}^{\mathrm{r}}$ Séminaire National sur le Laser et ses Applications, Alger 265.

[21] C. M. Tchen (1947) "Mean values and correlation problems connected with the motion of small particles suspended in a turbulent fluid" $\mathrm{Ph}$. D. Thesis Delft .

[22] J. O. Maxey and J. J. Riley (1983) "Equation of motion for a small rigid sphere in a non uniform flow" Physics Fluids 26, 883.

[23] B. Ruck (1990) "Einflu? der Tracerteilchengrö?e auf die Signalinformation in der Laser-Doppler-Anemometrie" Technisches Messen 57, 284.

[24] J. Quinard (1984) "Limites de stabilité et structures cellulaires dans les flammes de prémélange. Etude expérimentale" Thèse de Doctorat d'état, Université de Provence, France.

[25] G. Searby and P. Clavin (1986) "Weakly turbulent, wrinkled flames in premixed gases" Comb. Sci. 46, 167.

[26] J. Quinard, G. Searby and L. Boyer (1984) "The stability limits and critical size of structures in premixed flames Prog" Astronaut. Aeronaut. 95, 129.

[27] Kee R. J., Dixon-Lewis G., Warnatz J., Coltrin M. E. and Miller J. A. (1986) "The Chemkin Transport database" Sandia National Transport Laboratories Report Nº'SAN 86-8246.
[28] B. Renou, A. Boukhalfa, D. Puechberty and M. Trinité (1999) "Statistics of flame displacement speed from experimental study of freelypropagating premixed turbulent flames at various Lewis number" Proc. of the Med. Comb. Symp. Antalya, Turkie 